\title{
A Smart Wireless Ear-Worn Device for Cardiovascular and Sweat Parameter Monitoring During Physical Exercise: Design and Performance Results
}

\author{
Bruno Gil *, Salzitsa Anastasova and Guang Z. Yang \\ The Hamlyn Centre, Imperial College London, London SW7 2AZ, UK; \\ s.anastasova-ivanova@imperial.ac.uk (S.A.); g.z.yang@imperial.ac.uk (G.Z.Y.) \\ * Correspondence: b.gil-rosa@imperial.ac.uk; Tel.: +44-7470-311-442
}

Received: 31 January 2019; Accepted: 30 March 2019; Published: 4 April 2019

check for updates

\begin{abstract}
Wearable biomedical technology has gained much support lately as devices have become more affordable to the general public and they can easily interact with mobile phones and other platforms. The feasibility and accuracy of the data generated by these devices so as to replace the standard medical methods in use today is still under scrutiny. In this paper, we present an ear-worn device to measure cardiovascular and sweat parameters during physical exercise. ECG bipolar recordings capture the electric potential around both ears, whereas sweat rate is estimated by the impedance method over one segment of tissue closer to the left ear, complemented by the measurement of the lactate and $\mathrm{pH}$ levels using amperiometric and potentiometric sensors, respectively. Together with head acceleration, the acquired data is sent to a mobile phone via BLE, enabling extended periods of signal recording. Results obtained by the device have shown a SNR level of $18 \mathrm{~dB}$ for the ECG signal recorded around the ears, a THD value of $-20.46 \mathrm{~dB}$ for the excitation signal involved in impedance measurements, sweat conductivity of $0.08 \mathrm{~S} / \mathrm{m}$ at $1 \mathrm{kHz}$ and sensitivities of $50 \mathrm{mV} / \mathrm{pH}$ and $0.8 \mu \mathrm{A} / \mathrm{mM}$ for the $\mathrm{pH}$ and lactate acquisition channels, respectively. Testing of the device was performed in human subjects during indoors cycling with characteristic level changes.
\end{abstract}

Keywords: wearables; ear; electrocardiogram; sweat; lactate; pH; Bluetooth Low Energy

\section{Introduction}

Wearable biomedical technology has become ubiquitous in recent years, sparking a new research field in the area of IoT for healthcare applications [1]. Electronic miniaturization and advances in smart materials have allowed devices to be attached to the surface of the human body for measurement of physiological parameters, while consuming little amount of power and/or harvesting the energy from the body environment itself. Examples of such devices include glucose meters, biopotential monitors, motion trackers, odor sensors and UV detectors. The requirement for causing minimum discomfort and embarrassment to the user is guiding the design of the so-called wearables so as to be conformal with the body shape, in the form of flexible adhesive patches or incorporated into pieces of clothing and accessories. Many of these devices are already on the market in part to satisfy consumers' appetite for cutting-edge technology, but also to expand the research from the clinical environment to the comfort of home, workplace or sports venue [2].

Continuous monitoring of physiological variables is still largely limited to clinical environments and/or specialized laboratories that typically use bulky medical equipment certified for human trials. Such equipment requires galvanically-isolated power and data communication lines for the embedded electronics, as well as wiring connections to the recording spots over the body surface. 
Measurement of more than one signal modality involves recruiting additional equipment to detect the different signals simultaneously. Therefore, wearable device design must couple, in one end, with the acquisition of signals originated from different transduction mechanisms and, in the other end, employ low-power and low data-rate transfer protocols, like the Bluetooth Low Energy (BLE) for untethered applications that are battery-powered. Much like the case of the present study, where we committed ourselves to measure several signals related with the performance of physical exercise, including the assessment of cardiovascular, motion and sweat parameters, inside a small-form device developed for the head segment.

Body motion detection has been the target of a large portion of wearable technology developed in the past years, employing innovative ways to integrate accelerometers, gyroscopes and/or magnetometers into different body segments/joints and also taking into account the surrounding environment where the detection is performed [3]. In order to provide motion detection, we opted to include an accelerometer in the proposed device as the information originated by head acceleration can help complement the information derived from cardiovascular and sweat monitoring, by distinguishing if a sudden change in the latter parameters is related to exercise, as targeted by this study, or rather resulting from some pathological condition leading to movement restrictions, such as fever experienced during a systematic inflammatory response of the body. Regarding cardiovascular monitoring, measurement of the electrical activity of the heart has long been the preferential method to estimate heart rate (HR) and heart rate variability (HRV), useful to detect early signs of potential heart attack, arrhythmias and myocardial infection [4,5]. For exercise in particular, control of the individual effort by monitoring HR and HRV more accurately and frequently is important not only to avoid body injuries due to inadequate intensive strains but also to prevent sudden death, as the vast majority of exercises practiced in sport involve a short increase in the risk of heart attack. Modern ECG monitors limit the number of body leads in order to reduce dimensions and power consumption, acquiring the electrical signal preferentially over the chest and/or body limbs. In this study, we opted to acquire the ECG around the ears by using a single bipolar lead, hence preventing any wiring connections over the limbs (and chest) that can disturb exercise's performance. In the interface with the skin, novel ECG electrodes are also been developed to withstand higher temperatures, humidity levels and water immersion, occurring in some sport modalities. Moreover, special adhesives made of nanomaterials and epidermal electronics have been shown to produce less skin irritation, inasmuch like the E-textiles employed in the present study as an alternative to the traditional wet electrodes [6].

Sweat monitoring is another modality gaining much attention lately in wearable technology, which has application in the detection of early signs of skin dehydration and imbalances in the internal body fluids produced by intensive physical activities or some autonomic dysfunctions, such as hyperhidrosis and diabetes. Laboratory methods for measuring sweat are vast and include Miner's method [7], "wash-down" techniques [8], Parafilm patches [9] and impedance [10]. From all these methods, only impedance can be performed for extended periods of time outside laboratorial settings. However, impedance involves complex circuitry for wearables and it is neither completely immune to movement artifacts nor electrode placement. In this paper, we reduce the complexity in electronics by allowing a single excitation frequency in the lower spectrum $(1 \mathrm{kHz})$ where the conductivity effects dominate within the biological tissues, introducing also a new matrix made of electrodes to cover the tissues under analysis more uniformly. Such strategy avoids the problem related to electrode placement, while providing an area for sweat spreading (and dissipation), slowing down the process of channel saturation. In opposition to the approaches that use water vapor flow measurements [11] and humidity sensors printed on textiles [12], our solution employs a smaller and removable detection matrix, which minimizes the disturbance in the microclimate close to the skin where the sweat glands are located. Sweat also contains electrolytes that can be further analyzed such as $\mathrm{pH}$ and lactate for assessment of the athletes' performance or personal wellbeing [13]. $\mathrm{pH}$ is the analyte detected by means of a voltammeter transduction mechanism [14], wherein the concentration of protons in solution increases the voltage potential around an active electrode in reference to a bias level. The active $\mathrm{pH}$ electrode can 
be deposited using different techniques such as screen printing [15-17], dip casting, electrochemical deposition [18] and tailored according to the requirements using, in some cases, gold modified hydrogen ionosphere and plasticized PVC or graphene-polyurethane composites [19]. The typically high impedance of the electrode must be matched in the recording electronics by an ultra-low input bias current amplifier in order to reduce measurement errors introduced by the multiplication of the bias current of the amplifier by the electrode's impedance, masking the potential generated by the protons in solution (around $56 \mathrm{mV} /$ unit $\mathrm{pH}$ ). By its turn, lactate is recorded amperometrically by immobilizing lactate enzyme inside a sensing membrane and careful tailoring of the sensing layers to prevent unwanted interferences from other molecules in solution [20]. Lactate is then ionized and flows accordingly to the electric field imposed between a counter and reference electrodes, being detected through a working electrode that transforms this ionic flow into electrical current. Within this study, we deposited $\mathrm{pH}$ and lactate sensors over gold-platted electrodes located on a removable ear support, which contains also the electrodes for impedance and ECG. An additional membrane protects the chemical sensors from movement interferences, which constitutes a novelty when compared to the laboratory platforms found in the aforementioned literature, yet porous enough to allow sweat to flow. For the case of intense exercise leading to a faster saturation of the measurement channels, the ear support can easily be detached from the main circuitry board, washed and new sensors deposited again, assuring the reuse of the proposed technology.

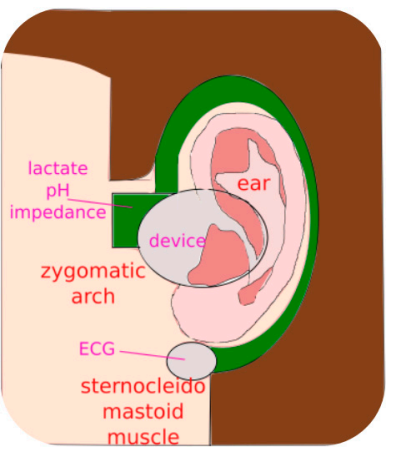

(a)

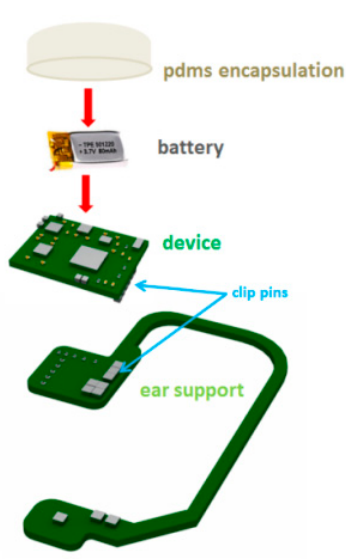

(b)

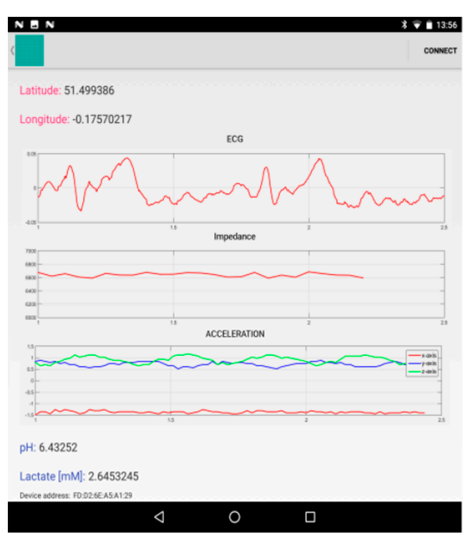

(c)

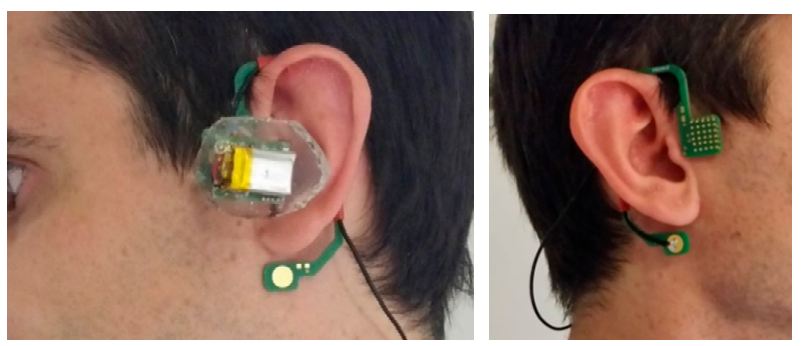

(d)

Figure 1. Prototype developed for measurement of cardiovascular, motion and sweat parameters around the ear, with total weight of $50 \mathrm{~g}$ : (a) Main anatomical features located around the left ear for acquisition of the ECG, impedance and chemical analytes through the skin; (b) Prototype assembly, involving device connection to an external battery, followed by PDMS encapsulation for protection of the electronics without compromising BLE transmissions and attachment to the ear support through clip pins; (c) Mobile phone's app developed to receive the BLE signals originated at the device; (d) Location of the assembled prototype over the left ear, while the right one holds the ear support only (although flipped) to contact the ECG electrode with the skin. 
From all the exposed above and in summary, in this paper we propose an ear-worn device to measure cardiovascular and sweat parameters during exercise with BLE connectivity. Unlike the approach followed by the authors of [21], the proposed device records bipolar ECG signals around the ears, whereas sweat rate is estimated by the impedance method. Recording of $\mathrm{pH}$ and lactate levels as well as head motion complements the specifications of the device, especially designed as an unobtrusive earpiece shown in Figure 1. Its location has been selected to facilitate access to the sweat accumulating in the upper part of the cheek (zygomatic arch) in the left side of the head, while the detection of the ECG signal occurs over the sternocleidomastoid muscle, hence avoiding the surrounding (non-conductive) bone areas. Our approach is to integrate multimodal sensing into a single device by making use of discrete off-the-shelf components. Another approaches use either large commercial chipsets or system-on-chip design into a custom-made smaller chip, which can integrate many functions and sensing capabilities by reconfiguring the basic analogue front-end blocks involved in the measurement of bio-potential, impedance and/or chemical analytes [22,23]. However, this latter approach has several disadvantages including longer design-to-production times and the inability to measure all modalities in simultaneous, besides requiring logical signals and reference clocks to select the desirable configuration, filter tuning and control that adds extra modulation noise originating from external complex digital circuitry. Moreover, multiplexing of input signals to meet the requirements of reconfigurable electronics is never a good option as it degrades the input impedance of the first amplifier on the electronic chain as seen directly by the source of the input signals, which must remain as higher as possible for detection of tiny bio-potential signals and/or $\mathrm{pH}$ level, as performed in this study.

\section{Materials and Methods}

This section describes in detail the electronic modules inside the wearable device, namely the ECG, impedance, $\mathrm{pH}$ and lactate measurement channels, as depicted in Figure 2a,b, for the layout of the printed circuit board and schematics, respectively. Electrode development for each of the aforementioned modalities is also thoroughly covered in this section. At the end, a description of the mobile phone app developed to receive the wireless signals finalizes the section.

\subsection{ECG Measurement Channel}

For ECG detection, a low noise instrumentation amplifier (INA333 from Texas Instruments, Dallas, TX, USA) was used with high amplification gain $(1000 \mathrm{~V} / \mathrm{V})$ to differentially amplify the signals around the upper part of the sternocleidomastoid muscle, as depicted in Figure 2b (ECG channel). The requirement for bipolar measurements has led to the use of a shielded wire to reduce noise interferences, routing the signal from the right ear to the left side of the head (device location). Each signal was picked-up by two electrodes made of woven conductive textile (Stretchy silver-plated nylon, Kitronik Ltd, Nottingham, UK), attached to the gold-platted pads on the ear support by silver conductive adhesive epoxy (8331-A, MG Chemicals Ltd, Surrey, BC, Canada), vide Figure 2d. Although these bare-gold pads can themselves pick-up the ECG signal, their flat surface is more prone to lose contact with the skin during motion, as opposed to textiles, which have a more porous and thick structure (larger contact area), yet soft enough not to cause skin bruises by constant rubbing or friction of the electrodes over the skin. After signal amplification, a $5^{\text {th }}$ - order lowpass filter with cut-off frequency $\left(f_{\text {cut }}\right)$ around $20 \mathrm{~Hz}$ was designed with standard operational amplifiers (LT1638, Analog Devices, Norwood, MA, USA) to reduce noise interferences, specially the capacitive coupling originating from the power grid line. Dimensioning of the resistors on the filter to meet the frequency requirement was calculated in accordance to Expression (1) by imposing the capacitors' values as $\mathbf{C}_{\mathbf{1}}=$ 
$4.7 \mu \mathrm{F}, \mathrm{C}_{2}=15 \mathrm{nF}, \mathrm{C}_{3}=33 \mathrm{nF}, \mathrm{C}_{4}=8.2 \mathrm{nF}$ and $\mathrm{C}_{5}=100 \mathrm{nF}$, and selecting the closest nominal values for the resistors as provided by the manufacturer, hence the approximations in the following formulas:

$$
\begin{gathered}
\mathrm{R}_{1}=\frac{1}{2 \pi \mathrm{f}_{\mathrm{cut}} \mathrm{C}_{1}} \simeq 1.8 \mathrm{k} \Omega, \quad \mathrm{R}_{2}=\frac{\mathrm{a}_{2} \mathrm{C}_{3}-\sqrt{\mathrm{a}_{2}^{2} \mathrm{C}_{3}^{2}-4 \mathrm{C}_{2} \mathrm{C}_{3}}}{4 \pi \mathrm{f}_{\mathrm{cut}} \mathrm{C}_{2} \mathrm{C}_{3}} \simeq 190 \mathrm{k} \Omega \quad\left(\mathrm{a}_{2}=1.62\right), \\
\mathrm{R}_{3}=\frac{\mathrm{a}_{2} \mathrm{C}_{3}+\sqrt{\mathrm{a}_{2}^{2} \mathrm{C}_{3}^{2}-4 \mathrm{C}_{2} \mathrm{C}_{3}}}{4 \pi \mathrm{f}_{\mathrm{cut}} \mathrm{C}_{2} \mathrm{C}_{3}} \simeq 670 \mathrm{k} \Omega, \quad \mathrm{R}_{4}=\frac{\mathrm{a}_{3} \mathrm{C}_{5}-\sqrt{\mathrm{a}_{3}^{2} \mathrm{C}_{5}^{2}-4 \mathrm{C}_{4} \mathrm{C}_{3}}}{4 \pi \mathrm{f}_{\mathrm{cut}} \mathrm{C}_{4} \mathrm{C}_{3}} \simeq 190 \mathrm{k} \Omega \quad\left(\mathrm{a}_{3}=0.62\right), \\
\mathrm{R}_{5}=\frac{\mathrm{a}_{3} \mathrm{C}_{5}+\sqrt{\mathrm{a}_{3}^{2} \mathrm{C}_{5}^{2}-4 \mathrm{C}_{4} \mathrm{C}_{3}}}{4 \pi \mathrm{f}_{\mathrm{cut}} \mathrm{C}_{4} \mathrm{C}_{3}} \simeq 2 \mathrm{M} \Omega
\end{gathered}
$$

The filter output was then digitalized with 10-bit of resolution by the embedded microcontroller (nRF51822, Nordic Semiconductor, Trondheim, Norway) at rates of 140 SPS for continuous segments of $30 \mathrm{~s}$, interleaved by the measurement of the impedance, $\mathrm{pH}$ and lactate signals, as described in the next sections.

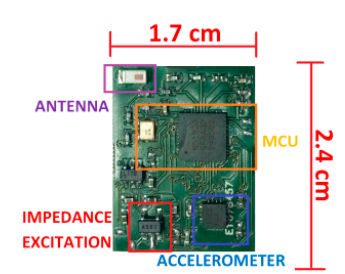

TOP

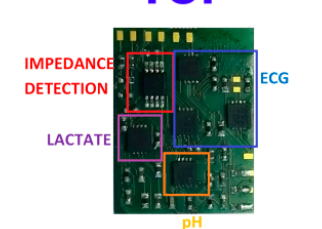

\section{BOTTOM}

(a)

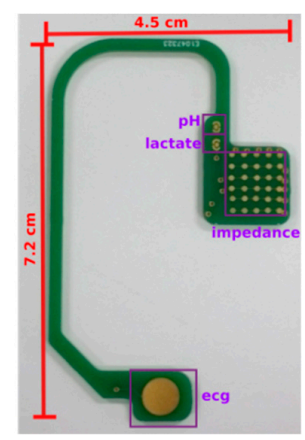

(c)

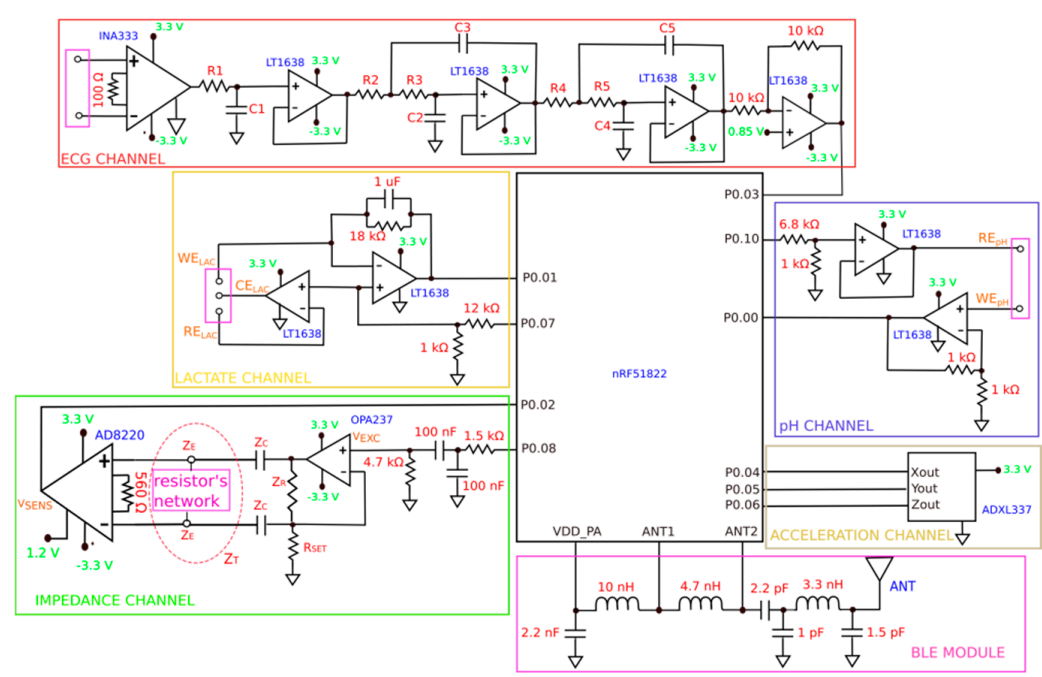

(b)

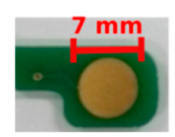

(d)

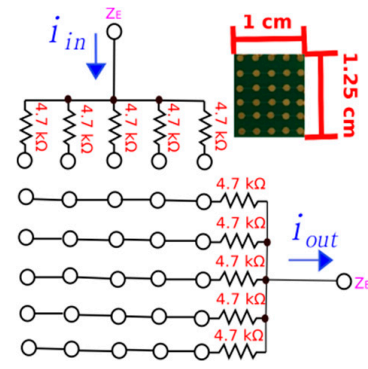

(e)

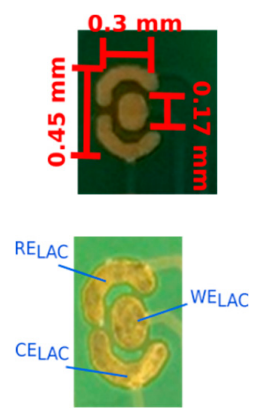

(f)

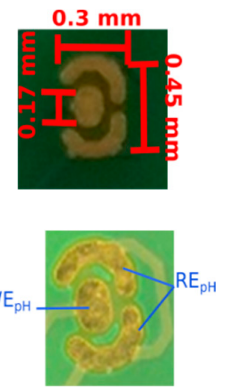

(g)

Figure 2. Different parts composing the ear-worn device: (a) Main electronic board with components assembled in the top and bottom layers; (b) Simplified electronic schematic covering the ECG, impedance, lactate and $\mathrm{pH}$ measurement channels; (c) Ear support developed to contact the electrodes and the sensors with the tissue around the ears; (d) ECG electrode covered with conductive textile; (e) Matrix of electrodes for impedance measurements, with equivalent resistors' network; (f) Lactate sensor deposition (WE $\mathrm{WEC}_{\mathrm{LAC}}$ ) with $\mathrm{RE}_{\mathrm{LAC}}$ and $\mathrm{CE}_{\mathrm{LAC}}$ electrodes, as captured by microscope (VHX-5000, Keyence, Milton Keynes, $\mathrm{UK})$; $(\mathrm{g}) \mathrm{pH}$ sensor deposition $\left(\mathrm{WE}_{\mathrm{pH}}\right)$ with single reference electrode $\left(\mathrm{RE}_{\mathrm{pH}}\right)$.

\subsection{Impedance Measurement Channel}

For impedance measurement of sweat, the bipolar configuration was employed that uses the same pair of electrodes for current injection and voltage detection. Electrodes were gold-plated not only to assure biocompatibility with the surrounding tissues, but also to lower impedance contact 
with the skin. A voltage-to-current converter (VI) was adopted in this study to transform a TTL signal generated by the microcontroller $(\mathrm{MCU})$ into a sinewave $\left(\mathrm{V}_{\mathrm{EXC}}\right)$ with a frequency of $1 \mathrm{kHz}$, by low-pass and high-pass signal filtering before VI conversion (OPA237, Texas Instruments, Dallas, TX, USA), as shown in Figure $2 b$ (impedance channel). The total circuit load $\left(Z_{L}\right)$ present in the feedback loop of the VI converter was determined beforehand in order to accommodate for possible impedance discrepancies generated over the interfaces device-electrodes and electrodes-tissue as:

$$
\frac{1}{Z_{L}}=\frac{1}{Z_{R}}+\frac{1}{2 Z_{C}+2 Z_{E}+Z_{T}} \Leftrightarrow Z_{L}=\frac{2 Z_{C}+2 Z_{E}+Z_{T}}{\frac{2 Z_{C}}{Z_{R}}+\frac{2 Z_{E}}{Z_{R}}+1}
$$

where $\mathbf{Z}_{\mathbf{R}}$ is the impedance of the closed-loop resistor $(560 \mathrm{k} \Omega), \mathbf{Z}_{\mathbf{C}}$ the impedance of the DC-blocking capacitors, $\mathbf{Z}_{\mathbf{E}}$ the impedance of the electrodes and $\mathbf{Z}_{\mathrm{T}}$ the impedance of the ear segment. By forcing $\mathbf{Z}_{\mathbf{R}}$ $>>\mathbf{Z}_{\mathbf{T}}, \mathbf{Z}_{\mathbf{E}} \rightarrow 0$ and $\mathbf{Z}_{\mathbf{C}} \rightarrow 0$ for $1 \mathrm{kHz}$ excitation, last expression yields $\mathbf{Z}_{\mathbf{L}} \simeq \mathbf{Z}_{\mathbf{T}}$. For a unitary voltage signal, the current-setting resistor $\left(\mathbf{R}_{\mathrm{SET}}\right)$ was chosen to $560 \mathrm{k} \Omega$ in order to impose a current limitation around $2 \mu \mathrm{A}$ to the living tissue. Within this condition, a lower value of impedance must be produced by $\mathbf{Z}_{\mathrm{T}}$ to prevent VI malfunctioning. By its turn, DC-blocking capacitors were added to the circuitry to prevent direct currents to flow into the head segment, thus avoiding electrolysis and the effects of permanent migration of free ions within the medium that occur during long-term recordings [24]. Limitation for AC current stimulation through the body is set to a magnitude of $100 \mu \mathrm{A}$, whereas skin impedances for humans are reported within the low kilo $\Omega$ range (lower spectrum), though a precise value is undefined as it depends on factors such as skin ageing, hydration level and tissue composition [24]. Regarding these two factors, we decided to produce an even smaller current so that the voltage drop produced by the skin could still be accommodated by the VI converter ( $\leq$ power supply level) in the eventuality of higher impedances present.

By its turn, for voltage detection, an instrumentation amplifier (AD8220, Analog Devices, Norwood, MA, USA, gain of $90 \mathrm{~V} / \mathrm{V}$ ) was selected to sense the signal developed across the electrodes ( $V_{\text {SENS }}$ ). The output of the amplifier is then digitalized at rates of $8 \mathrm{kSPS}$, yielding a total of 80 data points ( 8 points per period) employed in the estimation of the magnitude of $\mathbf{Z}_{\mathbf{T}}$ by the Discrete Fourier Transform (DFT) as:

$$
\operatorname{Real}\left\{X_{50}\right\}=\sum_{n=0}^{N} X_{n} \cos \left(-j 2 \pi \times 50 \frac{n}{N}\right), \quad \operatorname{Imag}\left\{X_{50}\right\}=\sum_{n=0}^{N} x_{n} \sin \left(-j 2 \pi \times 50 \frac{n}{N}\right)
$$

where $\mathbf{x}_{\mathbf{n}}$ is the 80-point sample vector, $\mathbf{n}$ the sample index, $\mathbf{N}$ the total number of samples and $\mathbf{X}_{\mathbf{5 0}}$ the $1 \mathrm{kHz}$ component. Conversion into amplitude is performed already inside the mobile phone's app by Expression (4) as it involves mathematical operations hard to implement inside the MCU:

$$
\left|Z_{\mathrm{T}}\right|=\operatorname{R}_{\mathrm{SET}} \frac{\sqrt{\operatorname{Real}\left\{X_{50}^{\mathrm{SENS}}\right\}^{2}+\operatorname{Imag}\left\{X_{50}^{\mathrm{SENS}}\right\}^{2}}}{\sqrt{\operatorname{Real}\left\{X_{50}^{\mathrm{EXC}}\right\}^{2}+\operatorname{Imag}\left\{X_{50}^{\mathrm{EXC}}\right\}^{2}}}
$$

An additional resistor network or matrix consisting of $6 \times 5$ millimeter-sized gold-plated electrodes was developed to detect more accurately the propagation of sweat around the left ear, as depicted in Figure 2e. This matrix of electrodes was projected to drive the imposed current through the area as soon as sweat enters in contact with the electrodes, lowering network's impedance. 


\section{3. $p H$ and Lactate Measurement Channels}

The $\mathrm{pH}$ measurement channel was developed by chemical deposition of a specially-designed $\mathrm{pH}$ sensor $\left(\mathrm{WE}_{\mathrm{pH}}\right)$ over the gold-platted electrodes in the ear support connected to the $\mathrm{pH}$ acquisition channel. The electronics for this channel employed a non-inverting amplifier (LT1638, Analog Devices, Norwood, MA, USA, gain of $2 \mathrm{~V} / \mathrm{V})$ with reference voltage $\left(\mathrm{RE}_{\mathrm{pH}}\right)$ set to $0.42 \mathrm{~V}$ by means of a voltage follower, as shown in Figure $2 \mathrm{~b}$ ( $\mathrm{pH}$ channel). By its turn, the lactate channel incorporated a specialized lactate sensor $\left(\mathrm{WE}_{\mathrm{LAC}}\right)$ connected to a transimpedance amplifier (current amplification of $18000 \mathrm{x}$ ) with counter $\left(\mathrm{CE}_{\mathrm{LAC}}\right)$ and reference $\left(\mathrm{RE}_{\mathrm{LAC}}\right)$ electrodes set to a potential of $0.25 \mathrm{~V}$, vide Figure $2 \mathrm{~b}$ (lactate channel). In terms of the fabrication process, the $\mathrm{pH}$ sensor was electrochemically deposited on an Ir wire forming IrOx metal-metal oxide $\mathrm{pH}$ sensor. The surface of the sensor was first electrochemically cleaned before membrane deposition. An intermediate layer of PEDOT was deposited to serve as a stabilising layer. Iridium oxide $\mathrm{pH}$ electrodes were then achieved through electrochemical oxidation during potential cycling between $0.2 \mathrm{~V}$ and $0.8 \mathrm{~V}$. The iridium oxide nanoparticle dispersion was made using $3.0 \mathrm{mM}$ aqueous $\mathrm{K}_{2} \mathrm{IrCl}_{6}$ solution adjusted to $\mathrm{pH} 10$ with $7 \%$ wt of aquous $\mathrm{NaOH}$. The resulting solution was cooled to room temperature and then adjusted to $\mathrm{pH} 2$ by rapidly adding $1 \mathrm{M} \mathrm{HNO}_{3}$ and stirred continuously for 40 minutes. For lactate, a dip coating procedure was followed for deposition of the sensing membrane. The electropolymerisation method for phenol red was used as an internal medium layer for the $\mathrm{WE}_{\mathrm{LAC}}$, by potential cycling between $+0.35 \mathrm{~V}$ and $+1 \mathrm{~V}$. The drop-casting solution contained enzyme $60 \mathrm{mg} / \mathrm{mL}$ glucose oxidase (GOx) from Aspergillus niger (Sigma Aldrich, St. Louis, $\mathrm{MO}, \mathrm{USA}$ ) and $30 \mathrm{mg} / \mathrm{mL}$ bovine serum albumin in $0.01 \mathrm{M}$ PBS. After enzyme immobilization, the sensor was additionally coated with a polyurethane film with 4 different concentrations ( 2 to $5 \%$ ) in order to extend its dynamic range. The developed sensors shown in Figure 2f,g were additionally covered with a protective membrane made of biocompatible polyurethane to prevent easy detachment from the ear support, yet porous enough for sweat to flow through and analytes to be detected by the sensors below.

\subsection{Mobile Phone's App and Power Consumption}

A graphical user interface was created in Android Studio to receive the data packets sent by the device via BLE, as shown in Figure 1c. The microcontroller inside device's electronics was selected in part because it contained a dedicated low-power $2.4 \mathrm{GHz}$ transceiver for BLE communication, which was easy to implement and tune in hardware by using some external resistors and capacitors, creating an RF matching circuitry (Pi-network) connected to a $50 \Omega$ single-ended antenna (2450AT18B100, Johanson Technology, Camarillo, CA, USA), see Figure 2b (BLE module). Transmissions were scheduled to occur every 100 miliseconds with data containing the samples acquired from each channel plus the information collected by a 3-axis accelerometer (ADXL337, Analog Devices, Norwood, MA, USA) with resolution of $0.01 \mathrm{~g}$ and range of $\pm 1.5 \mathrm{~g}$, depicted in Figure $2 \mathrm{~b}$ (acceleration channel). The data payload was set to 20 bytes per BLE packet, translated into a data communication rate of $1.6 \mathrm{kbps}$, with transmissions lasting for $10 \mathrm{~ms}$ and peak current consumption around $15 \mathrm{~mA}$. ECG samples are streamed continuously in $30 \mathrm{~s}$ segments, followed by $5 \mathrm{~s}$ segments containing, alternatively, the impedance, lactate and $\mathrm{pH}$ values. ECG data occupies 18 bytes (14 samples $\times 10$-bit) inside the packet, whereas the coefficients for impedance occupy 8 bytes, lactate $/ \mathrm{pH}$ values are transmitted within 10 bytes ( 8 samples with 10-bit resolution each) and the accelerometer information is compressed to 2 bytes only (5-bit resolution for each axis). An extra byte included in the BLE packet distinguishes between different modalities at the receptor's side. 
In terms of power consumption, the device presents two different profiles that translate the type of operation being performed by the MCU during its active cycle: data acquisition and data transmission. For the former, a current consumption of $15 \mathrm{~mA}$ was measured by a handheld multimeter (72-7730A, Tenma, Leeds, UK), while the latter consumed $25 \mathrm{~mA}(15 \mathrm{~mA}$ for communication plus an additional current of $10 \mathrm{~mA}$ drawn by the rest of the electronic components). Since the temporal extension of these operations is different, with acquisition occyping $90 \%$ of the cycle, the total power consumption is:

$$
\mathrm{t}_{\mathrm{ACQ}} \times \mathrm{I}_{\mathrm{ACQ}}+\mathrm{t}_{\mathrm{TRANS}} \times \mathrm{I}_{\mathrm{TRANS}}=16 \mathrm{mAh}
$$

where $\mathbf{t}_{\mathrm{ACQ}}$ and $\mathbf{t}_{\text {TRANS }}$ are the duration of the acquisition and transmission periods, respectively, whereas, $\mathbf{I}_{\mathrm{ACQ}}$ and $\mathbf{I}_{\text {TRANS }}$ represent the current consumption for the two operations. By employing an $80 \mathrm{mAh}$ rechargeable battery (501220, TPE New Energy, Shenzhen, China), the device can operate continuously for periods of $5 \mathrm{~h}$, enough to cover the physical activities performed in most of the sport modalities.

Finally, inside the graphical user interface, the ECG samples are further processed by means of a 10th-order lowpass filter $\left(\mathbf{f}_{\text {cut }}=10 \mathrm{~Hz}\right)$, whose finite impulse response for the $\mathbf{k}$ data sample is given by Expression (5). Filter coeficients were obtained by the Filter Design \& Analysis Toolbox from Matlab (Mathworks Inc., Natick, MA, USA) as part of a Gaussian window-type FIR with $\alpha$ set to 2.5:

$$
\begin{aligned}
y[k]=0.0855 x & {[k-1]+0.0886 x[k-2]+0.0913 x[k-3]+0.0932 x[k-4]+0.0943 x[k-5] } \\
& +0.0947 x[k-6]+0.0943 x[k-7]+0.0932 x[k-8]+0.0913 x[k-9] \\
& +0.0886 x[k-10]+0.0856 x[k-11]
\end{aligned}
$$

\section{Results}

This section is divided into the results obtained during the testing of the different channels of the device in laboratory, followed by experimentation with a real exercise. Whenever relevant, simulations of the propagation of the electric potential generated either by the heart or impedance channel will be also presented, as a complement to the results obtained by the device. Derivation of the mathematical formulation involved in these numerical simulations is described in Appendix A.

\subsection{ECG Channel Performance in Laboratory}

The single channel ECG was recorded in different electrode positions over the body as depicted in Figure 3a, namely, chest (sternum + 5th intercostal space), left shoulder/right leg, wrists and ears, hence providing a means of comparison between ECGs recorded at different electrode positions. The person remained in a seated position for the entire duration of the experiments, without performing any activity. These ECG signals originating at different locations were not acquired at the same time, but were synchronized a posteriori by their QRS complexes, as shown in Figure 3b. Voltage signals from different electronic stages within the device were also measured by an oscilloscope (MSO-X 3054A, 50 kSPS, Agilent Technologies, Santa Clara, CA, USA) for further analysis in the frequency domain, vide Figure 3c. Finally, the signal-to-noise ratio (SNR) of these signals was calculated in order to evaluate their quality (Figure 3d). In the computational domain, each signal was first low-pass filtered with cut-off frequency at $30 \mathrm{~Hz}$ (10th - order) to yield a desired noise-free signal, whereas the noise model was derived from the high-pass filtered version of the same signal with equal cut-off frequency and filter order. SNR values obtained this way correspond, therefore, to the ratio between the squared magnitudes of the $30 \mathrm{~Hz}$ ECG signal by its noise model. 


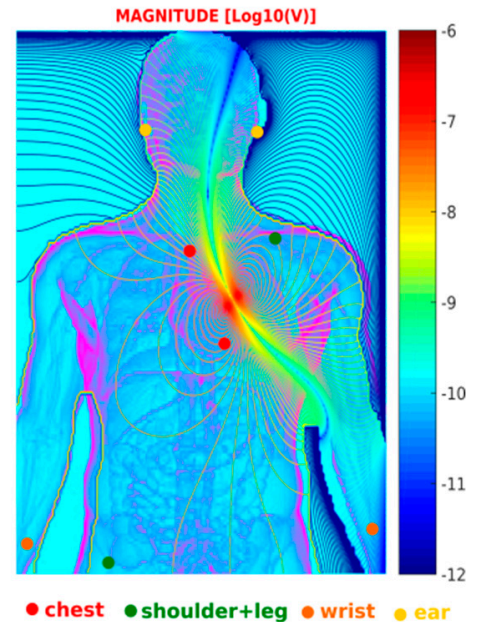

(a)

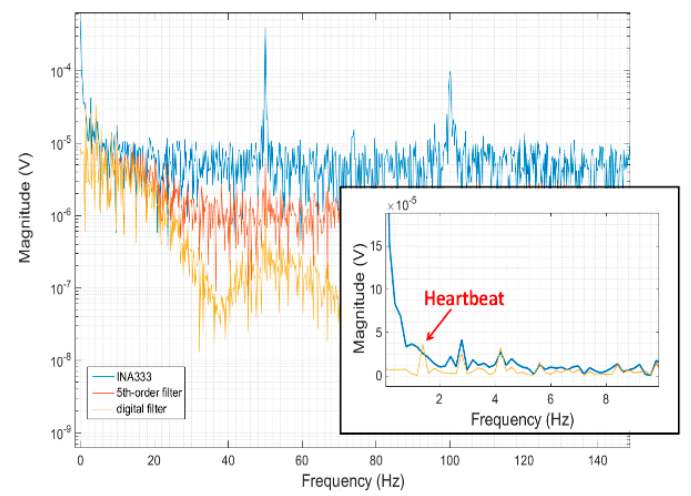

(c)

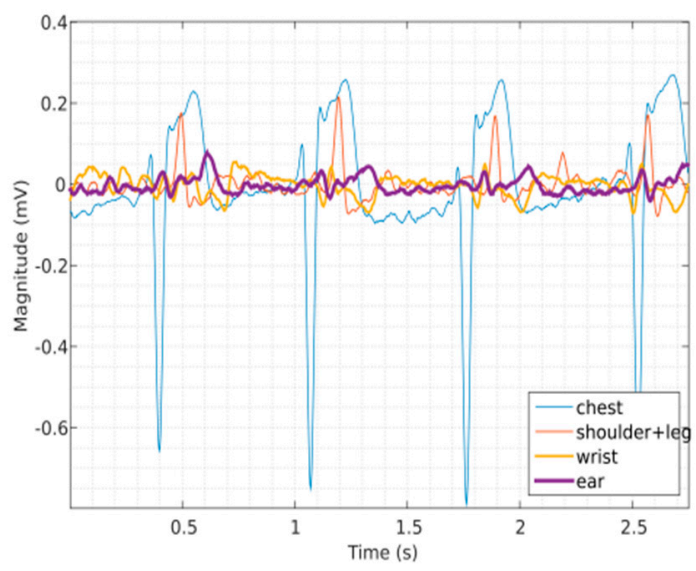

(b)

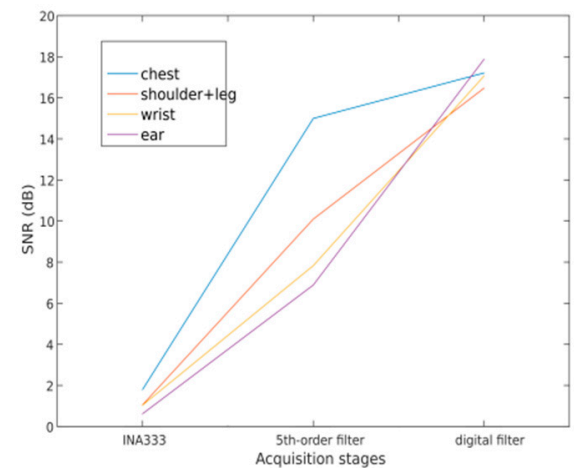

(d)

Figure 3. Testing of the ECG channel in different locations of the recording electrodes over the body: (a) ECG lead locations - chest, shoulder/leg, wrists and ears - superimposed over a depolarization map of the electric field generated by the heart; (b) ECG traces originating in the aforementioned body locations; (c) Spectrum for the ECG signal recorded around the ears and acquired at different stages in the electronic circuitry: amplification (INA333), analogue filter (5th-order) and digital filter, highlighting the noise reduction along the different stages and detail of the components closer to DC more related to ECG events; (d) Evolution of the SNR along the different electronic stages.

\subsection{Impedance Channel Performance in Laboratory}

The excitation signal produced by the device was acquired by the oscilloscope (rate of $100 \mathrm{kSPS})$ before entering VI conversion for the calculation of the total harmonic distortion value (THD), defined as the ratio between the first five harmonics of the signal and the fundamental, as shown in Figure $4 \mathrm{a}$. Then, the entire impedance channel was tested with a calibrated resistor's box (type 008-B, Cropico, Peterlee, UK) to evaluate the response of the DFT algorithm to standardized resistance values. In this scenario, the current-injecting and voltage-sensing electrodes were connected to the terminal inputs of the box, thereby forcing all the current to flow inside the resistor. The graphic obtained by this procedure is presented in Figure $4 \mathrm{~b}$ with respective linear fit. After calibration, the device was further tested with conductivity solutions (HI7030/31/33, Hanna Instruments, Woonsocket, RI, USA) as well as some body fluids. The samples were pipetted to a $0.6 \mathrm{~mL}$ centrifuge tube at room temperature and covered with a specially designed cap containing a $1 \times 2$ pin header connector $(2211 \mathrm{~S}-02 \mathrm{G}, 2.54 \mathrm{~mm}$ pitch, Multicomp, Leeds, UK) working as electrode and immersed to a volume of $0.5 \mathrm{~mL}$ inside the liquid (Figure 3c). Finally, the resistors' network in the ear support was experimented by pipetting a $0.1413 \mathrm{~S} / \mathrm{m}$ solution over the area covered by the matrix of electrodes and allowing the droplet to 
expand until all the electrodes were immersed (volume of $0.3 \mathrm{~mL}$ ), while recording the respective impedance signal (Figure 4d).

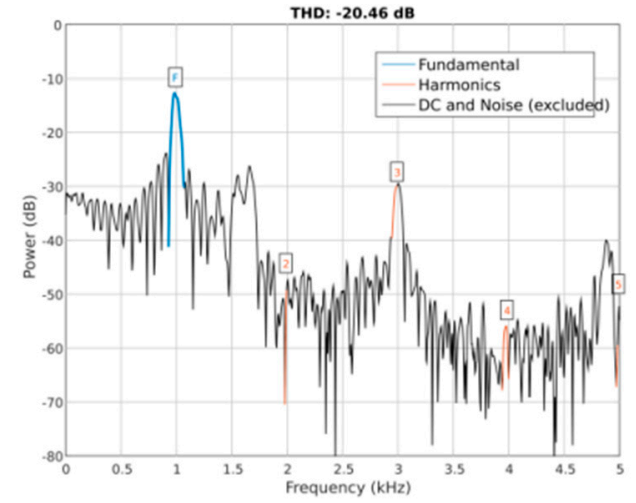

(a)

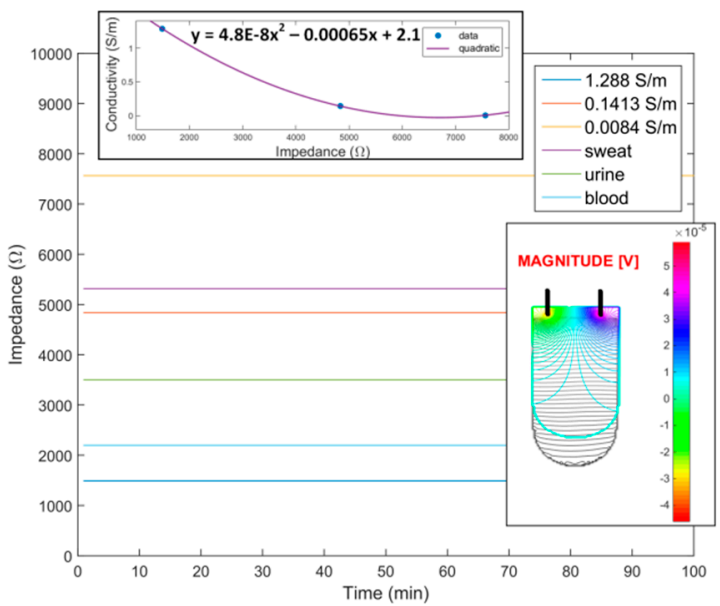

(c)

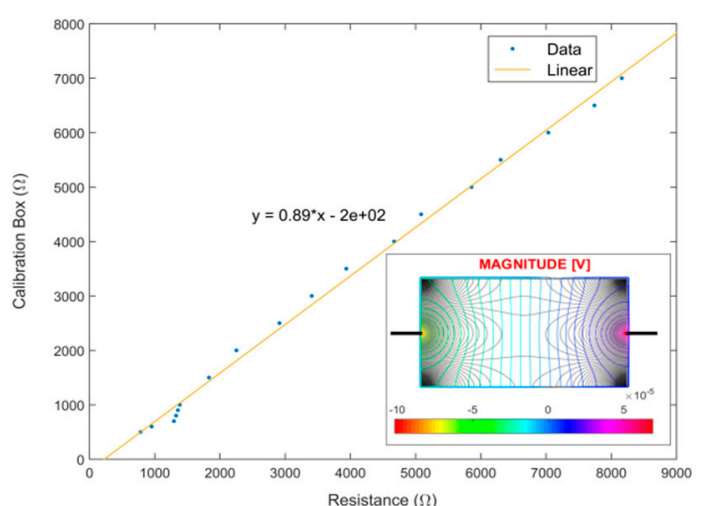

(b)

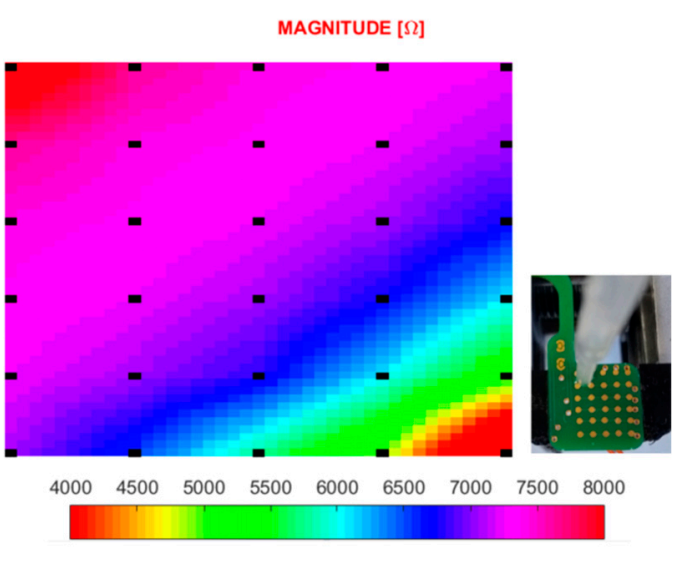

(d)

Figure 4. Characterization curves obtained for the impedance channel: (a) Power spectrum of the excitation signal, with separation between fundamental, harmonics and noise peaks; (b) Calibration of the channel by means of a calibration box, with a model for the propagation of the isocurrent (gray) and isopotential (color) lines presented in the inlet $\left(\sigma_{\text {resistor }}=1 \mathrm{~S} / \mathrm{m}\right) ;(\mathbf{c})$ Impedance value obtained for conductivity solutions and body fluids, with respective calibration curve and model for the propagation of the isocurrent and isopotential lines inside the centrifuge tube $\left(\sigma_{\text {tube }}=0.66 \mathrm{~S} / \mathrm{m}\right)$; (d) Impedance distribution map obtained while pipetting a $0.1413 \mathrm{~S} / \mathrm{m}$ solution along the resistors' network, from the electrodes located on the top left corner of the matrix to the bottom right ones.

\section{3. $\mathrm{pH}$ and Lactate Channels Performance in Laboratory}

The chemical sensors were tested after production with commercial solutions for $\mathrm{pH}$ with levels of 1, 4, 6, 7 and 9, followed by lactate with concentrations of 2, 9, 14, 20 and $24 \mathrm{mM}$. The calibration curves in terms of the voltage level per unit of the electrolyte are shown in Figure 5a,b, for $\mathrm{pH}$ and lactate, respectively, as obtained by a commercial electrochemical workstation (CHI600E, CH Instruments, Austin, TX, USA). Then, the same solutions were tried after attaching clean sensors to the ear support and connecting to the device. A droplet from each solution was pipetted over the respective electrode area with the signals obtained depicted in Figure 5c,d. 


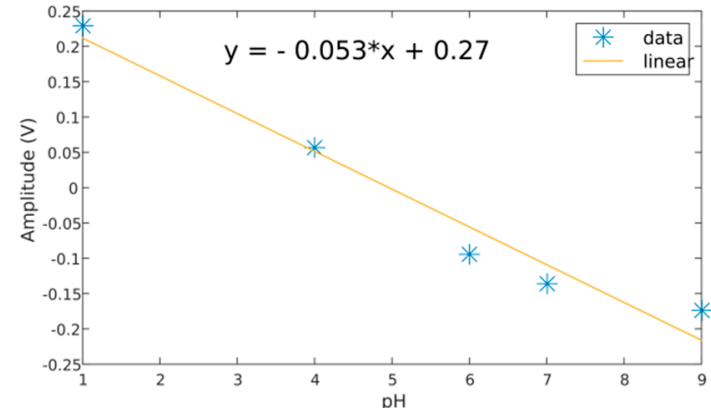

(a)

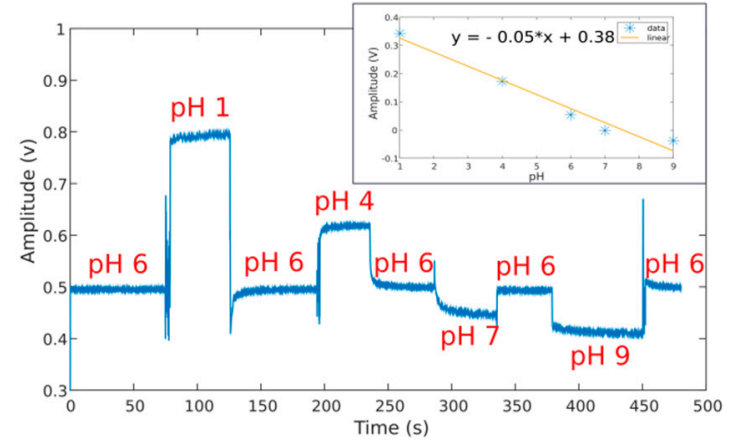

(c)

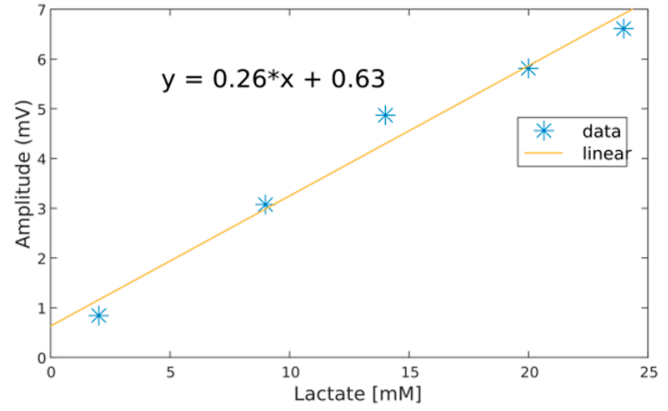

(b)

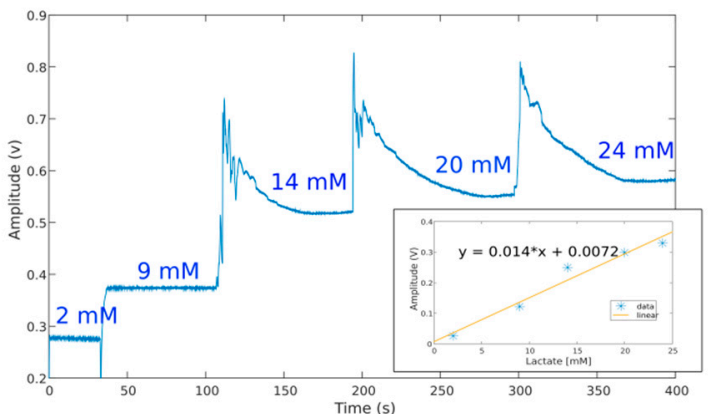

(d)

Figure 5. Characterization of the chemical sensors and measurement channels: (a) Calibration curve obtained for the $\mathrm{pH}$ sensor using a commercial electrochemical workstation; (b) Calibration curve obtained for the lactate sensor using the same workstation; (c) Temporal recording obtained by the $\mathrm{pH}$ measurement channel of the device and respective calibration curve for the tested solutions; (d) Temporal recordings and respective calibration curve for the lactate measurement channel while testing with different concentration solutions for the analyte.

\subsection{Testing of the Device in Real Exercise}

With the device fully tested and calibrated, indoors cycling exercise was performed by four volunteers using specialized equipment (Trainer 2013, Wattbike, Nottingham, UK). The trial lasted for a period of $20 \mathrm{~min}$, divided into a $10 \mathrm{~min}$ segment dedicated to exercise at a constant pace, followed by a 10 min period for recovery. An additional adhesive film (model 9780, 3M, Maplewood, MN, USA) was placed over the ear support and behind the ear to increase its fixation to the skin. BLE signals were acquired by a mobile phone (SM-G900F, Samsung, Seoul, Korea) running the developed app and located near the equipment. Ethical approval for this human trial was obtained from Imperial College London (ICREC 18IC4816) with each subject signing a consent form after being fully informed about the experiment. Figure 6 exhibits ECG and acceleration traces obtained at the beginning and middle of the exercise for the same volunteer, whereas Figure 7 shows the physiological signals measured for all subjects.

These signals were digitally processed offline by means of a 5-point moving average filter to eliminate some signal spurious from the recordings and smooth-out transitions between levels for better visualization. Heart rate measurement was performed by manually counting the number of QRS complexes as no algorithm was developed inside the graphical user interface to measure HR automatically. 


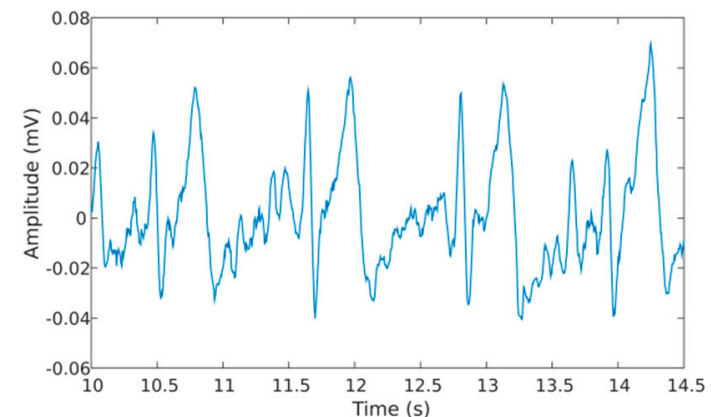

(a)

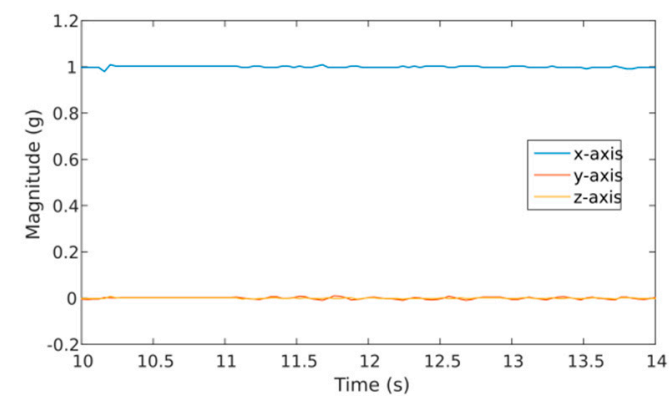

(c)

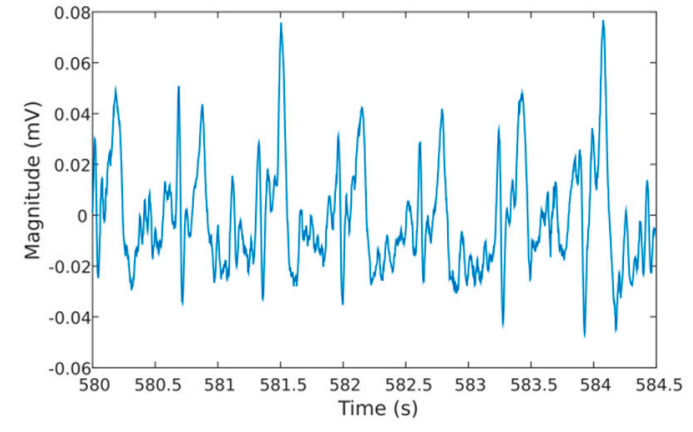

(b)

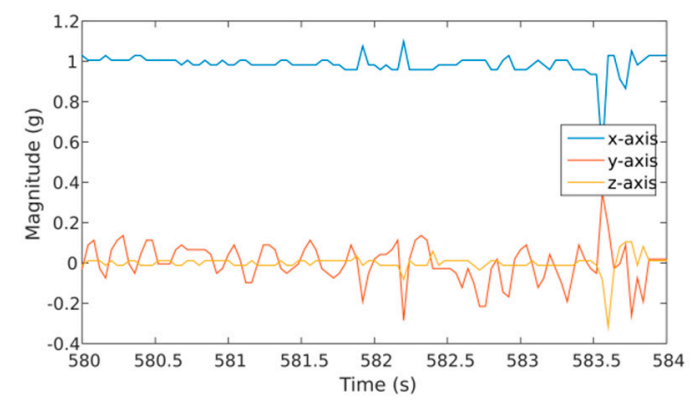

(d)

Figure 6. Temporal recordings obtained for one subject performing the indoors cycling exercise and wearing the proposed device: (a) ECG signal recorded at the beginning of the trial; (b) ECG obtained at the middle of the physical activity; (c) Acceleration registered at the beginning of the trial; (d) Acceleration obtained at the middle of the physical activity.

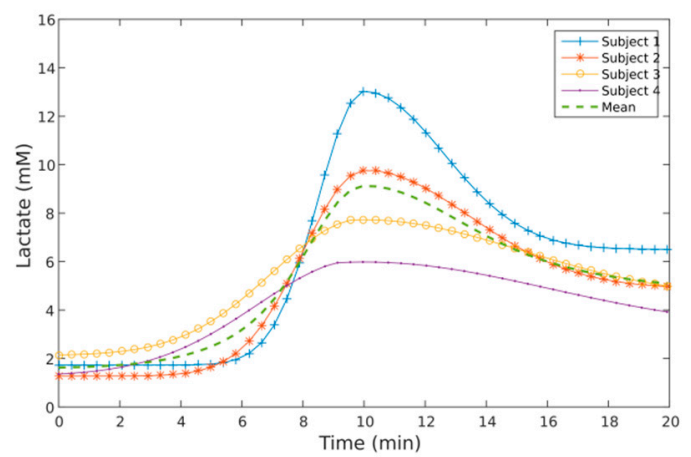

(a)

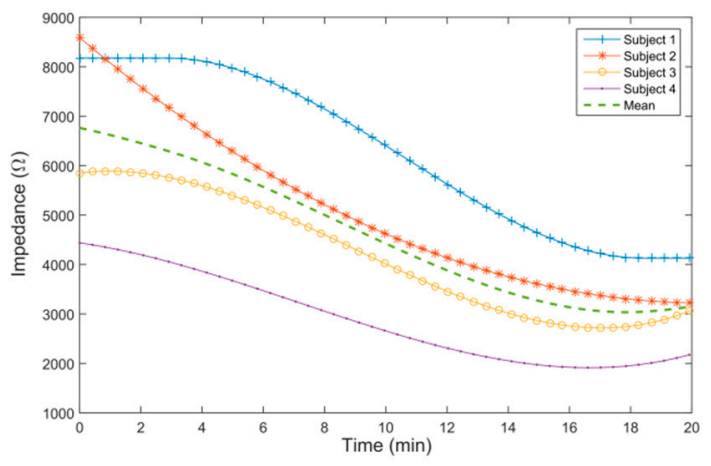

(c)

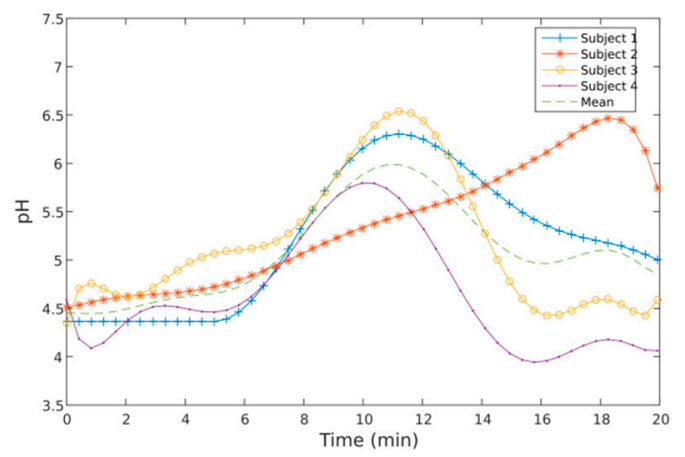

(b)

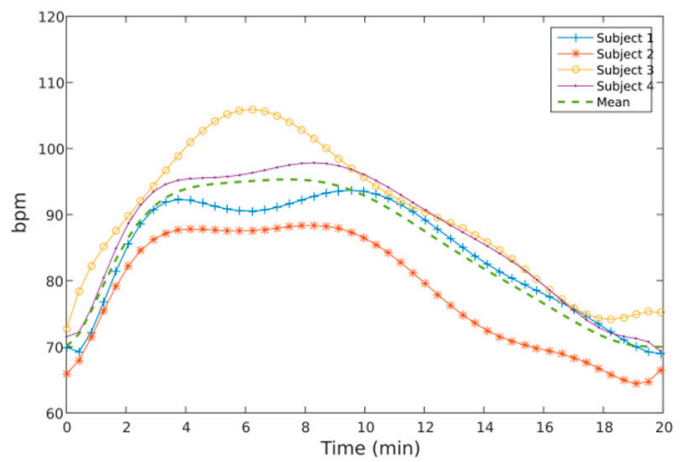

(d)

Figure 7. Variation of the physiological parameters during exercise (first $10 \mathrm{~min}$ ) followed by a recovery period, as obtained for all subjects: (a) Lactate; (b) $\mathrm{pH}$; (c) Impedance; (d) Heart rate. 


\section{Discussion}

The results obtained for the ECG channel have shown signals with higher SNR value for the chest lead, followed by the shoulder/leg, wrist and ear region, after amplification. The SNR levels obtained after amplification (INA333) are still relative small, which is reflected by the presence of a large amount of noise in the spectrum, especially from the $50 \mathrm{~Hz}$ power-grid (Figure 3c). Subsequent analogue and digital filtering has elevated the SNR to allow digitalization of the ECGs with moderate QRS definition, enough to estimate the heart beat even at the level of the ears, but with complete absence of the P-wave as experienced already by the authors of [21]. The measuring equipment employed to calculate SNR in this study also introduced additional noise and measurement errors to the values, though it was the only method available to access the target pins directly over the electronics. Moreover, defining the frequency components that belong exclusively to ECG events from those arising from other sources (including noise) is not a trivial process, if commercial and calibrated ECG recording equipment is not available for comparison. In any case, from Figure $3 b$, one can see that the signals acquired in the chest have the highest amplitudes and ears the lowest of all. This is further corroborated by the simulation model in Figure 3a, where the electric dipole propagates with higher intensity along the chest area, followed by the propagation into the limbs and finally to the head segment.

Regarding impedance, the excitation signal generated by the device yielded a THD value of $-20.46 \mathrm{~dB}$ (Figure 4a). This relative low value for THD is a consequence of filtering the square signal produced by the MCU with 1st-order filters only. This drawback was compensated by performing impedance calibrations using resistors and solutions with stable resistance values in the frequency domain, instead of relying directly on the result given by Equation (4). As the frequency of excitation is relatively slow $(1 \mathrm{kHz})$, the conductivity effects on the materials being tested prevail over permittivity and the dispersion effects. Conductivity effects are more frequency-independent and their influence can be understood by applying a simplified version of the Ohm's law. Within this regard, the loss in impedance magnitude is proportional to the decay induced by the influence of signal harmonics over the fundamental, whose real impedance value for materials and tissues can then be recovered by calibration. From the calibration graph in Figure $4 b$, it can be seen that the device responds to a range between $1 \mathrm{k} \Omega$ and $8 \mathrm{k} \Omega$, with a step resolution close to $10 \Omega$, a value obtained by considering the resolution of the acquisition channel (10-bit) and the interval of impedance values measured. The $10 \Omega$ resistor produces a voltage of $20 \mu \mathrm{V}$, which is already above the noise level of the differential amplifier employed in the acquisition channel $(14 \mathrm{nV} / \sqrt{ } \mathrm{Hz})$ within a $1 \mathrm{kHz}$ bandwidth $(14 \mu \mathrm{V})$. By its turn, measurement of the impedance value for solutions with different conductivities has allowed to obtain a fitting curve employed latter to detect the true conductivity level of some body fluids, independently of the geometry of the electrodes and sample container (Figure 4c). Conductivity levels measured by this method were $0.9 \mathrm{~S} / \mathrm{m}$ for blood, $0.08 \mathrm{~S} / \mathrm{m}$ for sweat and $0.4 \mathrm{~S} / \mathrm{m}$ for urine. Compared with literature reference (Table A1, Appendix A), these values are higher by almost a value of $0.2 \mathrm{~S} / \mathrm{m}$ (blood and urine only), though the experimental conditions were not the same between the current experiment and literature. For the simulation model in Figure $4 \mathrm{~b}$, the isocurrent lines for the resistor cross its entire extension, yielding potential lines symmetrically distributed between the recording electrodes, whereas in Figure 4c, some of the current lines escape from the top of the solution and their contribution to the voltage level detected is not accounted for, a fact that might explain the aforementioned discrepancies in conductivity.

For the chemical sensors, a sensitivity level of $53 \mathrm{mV} / \mathrm{pH}$ was measured for the fabricated $\mathrm{pH}$ sensor (Figure 5a), which declines to a level of $50 \mathrm{mV} / \mathrm{pH}$ with the electronic device (Figure $5 \mathrm{c}$ ). The bias current at the input terminal of the non-inverting amplifier can be responsible for this decline as well as the circuit board traces connecting the $\mathrm{pH}$ sensor to the amplifier, introducing further measurement errors. Nevertheless, the device with $\mathrm{pH}$ sensor attached was able to distinguish the $\mathrm{pH}$ from different solutions by producing proportional voltage levels. By its turn, for lactate, an increasing voltage level was obtained with the concentration of the analyte in solution for both the electrochemical workstation (Figure 5b) and proposed device (Figure 5d). For the latter, the sensibility is higher for 
the lower concentrations of lactate $(<20 \mathrm{mM})$ reflected in the higher voltage difference between signal baselines in Figure $5 \mathrm{~d}$. The complete lactate measurement channel saturates for concentrations close to $20 \mathrm{mM}$ as a consequence of the current amplification level set by device electronics. In the present conditions and attending to the fitting curve obtained, the voltage difference between tested solution $(\simeq 20 \mathrm{mM}$ ) is $0.25 \mathrm{~V}$, a value that divided by the resistor employed in the transimpedance amplifier $(18,000 \mathrm{k} \Omega)$ yields a detectable current level of $14 \mu \mathrm{A}$ or a standardized sensitivity level of $0.8 \mu \mathrm{A} / \mathrm{mM}$ for the sensor.

Finally, in what concerns the physical exercise, the parameters that experience faster changes are heart rate and impedance (Figure 7), with $\mathrm{pH}$ and lactate experiencing an abrupt rise at the middle of the indoors cycling event. Moreover, in the $10 \mathrm{~min}$ period allowed for recovery, these parameters do not return to their initial levels, in opposition to ECG, with all subjects going back to pre-exercise HR. By its turn, the impedance level never recovers completely from the decline proportionated by exercise, suggesting that some sort of micro fluidic channel must be included within the matrix to remove more efficiently the sweat during intensive physical activities or replace the ear support by a freshly new one in order to continue monitoring. At the end, the mean variation of the physiological parameters measured among all subjects during the trail are $\mathrm{pH}=5.10 \pm 1.54, \mathrm{LAC}=5.26 \pm 7.50 \mathrm{mM}$, $\mathrm{IMP}=4.59 \pm 3.73 \mathrm{k} \Omega$ and $\mathrm{HR}=85 \pm 25$.

\section{Conclusions}

An ear-worn device for cardiovascular and sweat monitoring has been experimentally tested under both laboratory and real exercise conditions. The need to confine the electronics into a small low-powered device has led to some simplifications in terms of circuitry implementation, namely the absence of higher-order filters to increase the THD of the excitation signal (impedance) and cascade amplifiers for the $\mathrm{pH}$ and lactate channels. Measurement of the phase for impedance was also not performed in this study as it remains unclear its influence in the course of the biological processes occurring inside tissues. The limitation on the acquisition rate of the internal ADC of the microcontroller ( $<10 \mathrm{kSPS})$ prevented the use of higher frequencies for impedance stimulation as well. By its turn, the absence of a driven right leg circuit for ECG had repercussions in the lower SNR values obtained in the first stage of signal amplification. With more robust electronics, we believe that it is still possible to detect all features of the ECG around the ears if higher ADC resolutions are employed (16-bit as commonly employed by front-end components in medical equipment) together with better shielding of device electronics and body segment.

To the best knowledge of the authors, this is the first study that combines the measurement of the cardiovascular performance with sweat parameters that has been fully integrated into a single ear-worn device. Control of the individual effort in physical activities is of paramount importance in sports in order to avoid injuries due to unadjusted intensive strains, a situation that the device can help prevent if overstrain is detected on the levels of the measured signals in real-time.

Finally, as future work, miniaturization of the device in terms of electronics can still be achieved externally by system-on-chip integration for every sensing modality independently or, internally, by means of an implantable device. In this latter scenario, bipolar ECG recordings are no longer feasible and HR estimation must be performed by means of optical measurements, such as pulse plethysmography over the earlobe. A flexible circuit board can also be designed for the ear support to adjust more naturally to the contour of the head and ears, though the capacitive substrates employed in the process may alter the impedance measurements. In the chemistry point-of-view, other analytes can also be detected in sweat by incorporation of new sensors with specialized membranes tailored to detect them. In this scenario, the specificity of each sensor must be individually evaluated against all the chemical species present in the medium, from each lactate is one of the most predominant analytes expelled during exercise. 
Author Contributions: Conceptualization, B.G. and S.A.; Methodology, B.G. and S.A.; Software, B.G.; Validation, B.G. and S.A.; Formal Analysis, B.G.; Investigation, B.G. and S.A.; Resources, G.Z.Y.; Data Curation, B.G.; Writing-Original Draft Preparation, B.G. and S.A.; Writing-Review \& Editing, B.G., S.A. and G.Z.Y.; Visualization, B.G.; Supervision, G.Z.Y.; Project Administration, G.Z.Y.; Funding Acquisition, G.Z.Y.

Funding: This research was funded by the Engineering and Physical Science Research Council under the project grants "EP/L014149/1" and "EP/R026092/1".

Acknowledgments: The authors would like to thank Dr. Benny Lo from the Hamlyn Centre, Imperial College London, to allow testing the device inside a sports facility equipped with sprint racer bike and all the work in obtaining the ethical approval for wearable device testing on human subjects.

Conflicts of Interest: The authors declare no conflict of interest.

\section{Appendix A}

The Finite Integration Technique (FIT) was used to create a simulation domain to evaluate the propagation of the electric potential generated by the heart (endogenous source) or external source (impedance). FIT has long been used to implement Maxwell's equations on electromagnetism into a discrete formulation suitable for computers, while dividing the domain into a limited number of regular cell-elements $[25,26]$. In this study, the domain was divided into $\mathrm{N}=40000$ rectangular elements. The calculation of the electric potential inside the domain has followed the principles dictated by the Electrical Impedance Tomography (EIT) by knowing, in advance, the distribution of the conductivity $(\sigma)$ of body tissues within the human body (coronal slice) and $\sigma$ for the materials inside the resistor's model or centrifuge tube, in accordance to Table A1. Then, the equations for EIT were implemented with the same mathematical simplifications as performed in $[27,28]$, by assuming an electrostatic condition for Ampere's law and the irrotational nature of the electric field in the harmonic regime of stimulation, thus obtaining:

$$
\nabla \cdot \sigma \nabla_{\Phi}=0
$$

where $\nabla$. is the divergence operator and $\nabla_{\boldsymbol{\Phi}}$ the gradient of the electric potential $\boldsymbol{\Phi}$. In the elements defining the electric stimulus, conditions for neutral electric charges were imposed, in the form of source and sink points, where the electric current could flow in and out, as given by Equation (A2):

$$
\Phi^{\text {in }}=-\Phi^{\text {out }}
$$

The Maxwell-Grid equations just presented were implemented computationally as a system of the form $\mathbf{A} \mathbf{u}=\mathbf{b}$, where $\mathbf{A} \in R^{N x N}$ represents the product of the topological operators with the conductivity map, $\mathbf{b} \in R^{N x 1}$ an all-zero column vector except in the pair of elements defining the stimulus (electrodes) and $\mathbf{u} \in R^{N x 1}$ the solution of the system in terms of $\boldsymbol{\Phi}$. Inversion of the system $\mathbf{u}=$ $\mathbf{A}^{-1} \mathbf{b}$ was achieved without pre-conditioning the highly sparse matrix $\mathbf{A}$, with the system converging to a solution within a couple of seconds of processing, this using an Intel Core i7-4770 $(3.4 \mathrm{GHz})$ processor with $16 \mathrm{~GB}$ of system RAM at the disposal of the numerical routine.

Table A1. Conductivity values for some biological tissues and fluids at $1 \mathrm{KHz}$ [24].

\begin{tabular}{cccc}
\hline Tissue & $\boldsymbol{\sigma}(\mathbf{S} / \mathbf{m})$ & Fluid & $\boldsymbol{\sigma}(\mathbf{S} / \mathbf{m})$ \\
\hline Skin & 0.001 & Blood & 0.7 \\
Bone & 0.02 & Urine & 0.2 \\
Heart & 0.1 & Fat & 0.03 \\
Lung & 0.03 & Air $^{1}$ & $1 \times 10^{-6}$ \\
Muscle & 0.3 & &
\end{tabular}

${ }^{1}$ The conductivity of the air is null but, in order to prevent numerical instabilities, a small value was allocated. 


\section{References}

1. Yang, G.Z. Implantable Sensors and Systems: From Theory to Practice; Springer International Publishing: Cham, Switzerland, 2018.

2. Athavale, Y.; Krishan, S. Biosignal monitoring using wearables: Observations and opportunities. Biomed. Signal Process. Control 2017, 38, 22-23. [CrossRef]

3. Di Rienzo, M.; Vaini, E.; Lombardi, P. Development of a smart garment for the assessment of cardiac mechanical performance and other vital signs during sleep in microgravity. Sens. Actuator A Phys. 2018, 274, 19-27. [CrossRef]

4. Sun, F.; Yi, C.; Li, W.; Li, Y. A wearable H-shirt for exercise ECG monitoring and individual lactate threshold computing. Comput. Ind. 2017, 92-93, 1-11. [CrossRef]

5. Li, S.H.; Lin, B.S.; Wang, C.A.; Yang, C.T.; Lin, B.S. Design of wearable and wireless multi-parameter monitoring system for evaluating cardiopulmonary function. Med. Eng. Phys. 2017, 47, 144-150. [CrossRef]

6. Guzik, P.; Malik, M. ECG by mobile technologies. J. Electrocardiol. 2016, 49, 894-901. [CrossRef]

7. Sato, K.T.; Richardson, A.; Timm, D.E.; Sato, K. One-step iodine starch method for direct visualization of sweating. Am. J. Med. Sci. 1988, 295, 528-531. [CrossRef]

8. Lemon, P.W.; Yarasheski, K.E. Feasibility of sweat collection by whole body washdown in moderate to high humidity environments. Int. J. Sports Med. 1985, 6, 41-43. [CrossRef]

9. Shirreffs, S.M.; Maughan, R.J. Whole body sweat collection in humans: An improved method with preliminary data on electrolyte content. J. Appl. Physiol. 1997, 82, 336-341. [CrossRef]

10. Medrano, G.; Beckmann, L.; Zimmermann, N.; Grundmann, T.; Gries, T.; Leonhardt, S. Bioimpedance spectroscopy with textile electrodes for a continuous monitoring application. In Proceedings of the 4th International Workshop on Wearable and Implantable Body Sensor Networks, Aachen, Germany, 26-28 March 2007; pp. 23-28.

11. Coyle, S.; Lau, K.T.; Moyna, N.; O’Gorman, D.; Diamond, D.; Francesco, F.D.; Constanzo, D.; Salvo, P.; Trivella, M.G.; Rossi, D.E.; et al. BIOTEX-Biosensing Textiles for Personalised Healthcare Management. IEEE Trans. Inf. Technol. B 2010, 14, 364-370. [CrossRef]

12. Salvo, P.; Pingitore, A.; Barbini, A.; Francesco, F.D. A wearable sweat rate sensor to monitor the athletes' performance during training. Sci. Sport 2018, 33, 51-58. [CrossRef]

13. Lopez, F.B.; Coyle, S.; Byrne, R.; Smeaton, A.; O'Connor, N.E.; Diamond, D. Pump less Wearable Microfluidic Device for Real Time pH Sweat Monitoring. Procedia Chem. 2009, 1, 1103-1106. [CrossRef]

14. McCaul, M.; Glennon, T.; Diamond, D. Challenges and opportunities in wearable technology for biomedical analysis in sweat. Curr. Opin. Electrochem. 2017, 3, 46-50. [CrossRef]

15. Kassal, P.; Steinberg, M.D.; Steinberg, I.M. Wireless chemical sensors and biosensors: A review. Sens. Actuator B Chem. 2018, 266, 228-245. [CrossRef]

16. Anastasova, S.; Mattinen, U.; Radu, A.; Bobacka, J.; Lewenstam, A.; Danielewski, M.; Diamond, D. Development of miniature all-solid contact potentiometric sensing system. Sens. Actuator B Chem. 2010, 146, 199-205. [CrossRef]

17. Anastasova, S.; Radu, A.; Matzeu, G.; Zuliania, C.; Mattinen, U.; Bobacka, J.; Diamond, D. Disposable solid-contact ion-selective electrodes for environmental monitoring of lead with ppb limit-of-detection. Electrochim. Acta 2012, 73, 93-97. [CrossRef]

18. Anastasova, S.; Crewther, B.; Bembnowicz, P.; Curto, V.; Ip, H.M.; Rosa, B.; Yang, G.Z. A wearable multisensing patch for continuous sweat monitoring. Biosens. Bioelectron. 2017, 93, 139-145. [CrossRef] [PubMed]

19. Dang, W.; Manjakkal, L.; Navaraj, W.T.; Lorenzelli, L.; Vinciguerra, V.; Dahiya, R. Stretchable wireless system for sweat pH monitoring. Biosens. Bioelectron. 2018, 107, 192-202. [CrossRef] [PubMed]

20. Spehar-Deleze, A.M.; Anastasova, S.; Vadgama, P. Electropolymerised Phenolic Films as Internal Barriers for Oxidase Enzyme Biosensors. Electroanalysis 2014, 26, 1335-1344. [CrossRef]

21. He, D.H.; Winokur, E.S.; Sodini, C.G. An Ear-Worn Vital Signs Monitor. IEEE Trans. Biomed. Eng. 2015, 62, 2547-2552. [CrossRef] [PubMed]

22. Kim, J.; Ko, H. Reconfigurable Multiparameter Biosignal Acquisition SoC for Low Power Wearable Platform. Sensors 2016, 16, 2002. [CrossRef] [PubMed] 
23. Kim, I.; Lobo, R.; Homer, J.; Bhagat, Y.A. Multimodal analog front end for wearable bio-sensors. IEEE Sens. J. 2016, 16, 8784-8791. [CrossRef]

24. Grimmes, S.; Martinsen, O.G. Bioimpedance and Bioelectricity Basics, 2nd ed.; Elsevier Ltd.: Amsterdam, The Netherlands, 2008.

25. Clemens, M.; Weiland, T. Discrete Electromagnetism with the Finite Integration Technique. J. Electromagn. Wave 2001, 15, 78-80. [CrossRef]

26. Schuhmann, R.; Weiland, T. Conservation of Discrete Energy and related laws in the Finite Integration Technique. J. Electromagn. Wave 2001, 15, 477-478. [CrossRef]

27. Bayford, R.H. Bioimpedance Tomography (Electrical Impedance Tomography). Annu. Rev. Biomed. Eng. 2006, 8, 63-91. [CrossRef] [PubMed]

28. Kim, K.J.; Kang, S.I.; Kim, S.; Lee, Y.L.; Vauhkonen, M. Dynamic Electrical Impedance Tomography with Known Internal Structures. Inverse Probl. Eng. 2003, 11, 1-19. [CrossRef]

(C) 2019 by the authors. Licensee MDPI, Basel, Switzerland. This article is an open access article distributed under the terms and conditions of the Creative Commons Attribution (CC BY) license (http:/ / creativecommons.org/licenses/by/4.0/). 